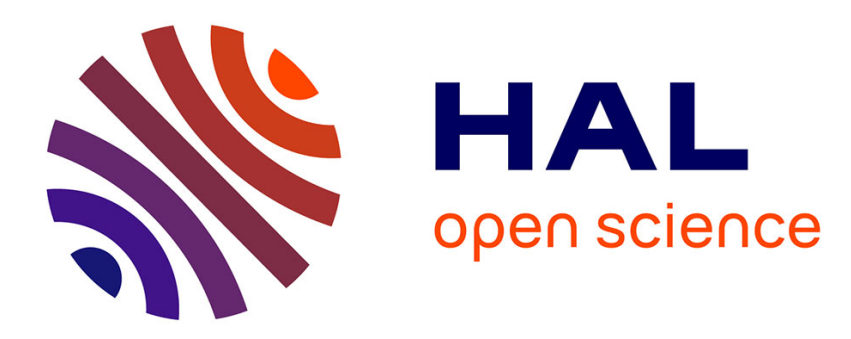

\title{
Une innovation sociale dans les plis du droit \\ Jean-Yves Trépos
}

\section{To cite this version:}

Jean-Yves Trépos. Une innovation sociale dans les plis du droit: Regard sur un dispositif d'activation des pratiques judiciaires, policières et sociales. Champ Pénal, 2017, XIV, 10.4000/champpenal.9567. hal-02979355

\section{HAL Id: hal-02979355 \\ https://hal.univ-lorraine.fr/hal-02979355}

Submitted on 27 Oct 2020

HAL is a multi-disciplinary open access archive for the deposit and dissemination of scientific research documents, whether they are published or not. The documents may come from teaching and research institutions in France or abroad, or from public or private research centers.
L'archive ouverte pluridisciplinaire HAL, est destinée au dépôt et à la diffusion de documents scientifiques de niveau recherche, publiés ou non, émanant des établissements d'enseignement et de recherche français ou étrangers, des laboratoires publics ou privés. 


\section{Une innovation sociale dans les plis du droit Regard sur un dispositif d'activation des pratiques judiciaires, policières et sociales}

Nous allons nous intéresser à une situation en trompe-l'œil. A première vue, la construction des violences domestiques comme problème public (les «violences conjugales et intrafamiliales » en tant que scène d'intervention) est à peu près achevée en Europe : la pluralité des institutions habilitées à s'en saisir via des dispositifs de plus en plus diversifiés laisse penser que les sociétés européennes disposent aujourd'hui des moyens d'action adéquats et des professionnels compétents. Et pourtant, lorsque les intervenants spécialisés, principaux animateurs de cette Scène soulignent, qui sa frustration, qui sa colère, ce n'est pas tant au regard des moyens dont ils disposent (certes insuffisants à leurs yeux) qu'à l'égard des attitudes et comportements de beaucoup de ceux qui occupent des positions décisives - au parquet, dans la police et dans les institutions d'accueil - pour une mise en œuvre efficace de ces dispositifs.

Autrement dit, ils semblent attendre moins une transformation de l'équipement juridique qu'une évolution de la pratique judiciaire; moins un affichage renforcé des services ou personnes spécialisés au sein de la police qu'une réelle incorporation de cette attention spécifique au travail ordinaire des policiers ; moins une augmentation, dans les institutions d'accueil, des signalements de violences subies par des mères isolées, qu'une capacité à s'en saisir sur place pour une réponse d'urgence. Dès lors, ceux qui souhaitent intensifier les transformations de la prise en charge des violences conjugales - parce qu'ils (plus souvent : elles) ont déjà été à l'initiative des premiers dispositifs - entreprennent de mobiliser sans délai, avec les moyens existants, tous ces représentant de l'ordre public et social qui se satisfont de leurs seconds rôles alors même qu'ils sont, comme le dit la métaphore militaire, « en première ligne ».

Mais doit-on s'en tenir à cette présentation flatteuse d'une transformation bottom up, qui montrerait une bonne volonté militante capable de secouer un mastodonte ralenti ? Peut-on vraiment imaginer que des professionnels épuisés par leurs petites victoires contre l'indifférence et le déni se lanceraient dans une aventure encore plus risquée s'ils ne décelaient pas des occasions favorables, voire des mouvements internes aux mondes judiciaire et policier? Allons plus loin: ne seraient-ce pas des transformations des rationalités du système répressif qui rendraient envisageables - top down - de nouvelles formes de prise en charge sociales? Le modèle adéquat pour calibrer notre attention sur ce problème est très probablement dialectique (les transformations de rationalités seraient alors accélérées et/ou déviées par les croisades morales qu'elles auraient rendu possibles).

On peut supposer que les différents protagonistes de ce nouveau système s'accordent pour l'installer parce qu'ils composent, dans l'action, des visions du monde compatibles (selon la théorie d'Apostel, résumée par Vidal, 2008 : des valeurs, des conceptions de l'action, de la temporalité et de la vérité), mais cela ne définit qu'un terrain favorable : encore faut-il savoir mettre en culture cet espace, rendre une Cause audible. Comment ces bâtisseurs d'une nouvelle variante de l'intérêt public peuvent-ils lisser les plis du droit sans froisser des magistrats dont le rôle reste majeur, se défaire de l'emprise de l'approche répressive tout en offrant des prises de sécurisation à leur population de référence, s'émanciper de la psychologisation de la maltraitance sans minimiser la singularité des souffrances ou la saillance des comorbidités?

On l'entrevoit : ils doivent parvenir à faire partager leur travail de problématisation pour espérer transformer des intervenants dispersés en partenaires. C'est l'ensemble de ce processus, repéré à partir de deux phases majeures (la problématisation et l'enrôlement) qui 
occupera l'essentiel de mon propos dans ma deuxième et ma troisième partie. Je ne m'attaque donc pas de manière détaillée et frontale au «pourquoi » d'un nouveau système d'interaction entre le psycho-social et le judiciaire (l'espace théorique de la réponse est toutefois donné dans une première partie), mais j'analyse surtout le «comment» de sa mise en place. Il n'est pas interdit de penser que l'attention ainsi portée aux démêlés de l'innovation sociale apportera à l'état pratique des réponses consistantes aux interrogations initiales sur les conflits de rationalité.

\section{Une dialectique de l'initiative juridique et de l'innovation sociale}

D'un point de vue synchronique, on pourrait dresser une première configuration de la situation étudiée ici: les intervenants sociaux sont confrontés simultanément à une construction politico-juridique de l'espace domestique faite de textes superposés présentant de nombreuses zones d'ombre (assimilables à des "plis ») et à un exercice répressif marqué par des hiérarchies de fait en termes d'urgence et d'attribution de responsabilité (exerçant une « emprise », par inertie ou par simplification abusive, sur tous les participants à l'action). Pour y répondre, ils cherchent à équiper cette situation à l'aide d'outils suffisamment stables (des «prises » solides), avec pour horizon de référence une conception générale des valeurs, de l'action, de la temporalité et de la vérité (une vision du monde) qui sont pour eux autant de « repères $\|^{1}$.

Mais, considérées dans la perspective d'un déroulement temporel, c'est sans doute à une image moins démiurgique qu'il faudrait avoir recours pour configurer ces entreprises et saisir la relation dialectique qu'elles entretiennent avec le tissu social. Ces initiatives doivent sans doute leur existence à des transformations lentes de l'appareil juridique (désignation globalisatrice qui inclut la production de droit et la mise en œuvre judiciaire au sein du legal complex) et à sa perméabilité relative (par capillarité ou par filtrage entre normes juridiques et normes sociales) aux actions incitatives de mouvements sociaux, d'où émergent des territoires à catégoriser. C'est sur ce fond que les innovateurs suivis ici élaborent leurs schémas, bâtissent leurs alliances et rendent leurs idées acceptables. Mais parce que ce sont des innovateurs et pas seulement des inventeurs, nous ne pouvons pas nous contenter de les décrire et ignorer ceux qu'ils parviennent à mettre en mouvement, ce qui nécessiterait de contextualiser les mouvements qu'ils suscitent dans cet espace social. Pour autant, entreprendre de réunir tous les éléments sociétaux (notamment: construction politique, pressions citoyennes) engagés dans la production d'un «contexte » serait nettement hors de notre portée dans le cadre d'un article qui revendique d'éclairer une initiative historiquement et géographiquement située. C'est pourquoi s'en tenir à une contextualisation juridique paraît être une ambition plus raisonnable : "régler la mire " sur la situation du droit concernant les violences domestiques au regard de l'évolution du droit en général pour donner le cadre temporel dans lequel émergent des actions innovantes et qu'elles contribuent à modifier par contrecoup.

\subsection{Trois moments de temporalités agrégées}

De ce processus dialectique, on ne retiendra que les trois moments les plus saillants de temporalités imbriquées. Le premier pourrait être dit macrosocial : il invite à soumettre un constat (les changements observables au sein des politiques pénales et des pratiques

\footnotetext{
${ }^{1}$ Ce registre métaphoriques (pli, prise, emprise, repères) prend appui sur les conceptualisations élaborées par Francis Chateauraynaud et son équipe (Chateauraynaud, 2011), qui offre l'avantage d'être d'un outil de présentation ayant une forte charge intuitive.
} 
judiciaires, sans omettre leur amont et leur aval policiers) concernant les violences conjugales à des hypothèses sur les évolutions du droit (notamment pénal). Le deuxième, méso-social, invite à mesurer la portée perturbatrice de l'activisme associatif lorsqu'il s'attaque à la trop grande abstraction des mécanismes juridiques macrosociaux supposés traiter ce problème social. Le troisième moment, méso-social lui aussi, apparaît comme une synthèse des deux précédents : de la rencontre des innovations associatives et des évolutions lentes du système pénal, naissent des dispositifs de travail qui laissent entrevoir des avancées significatives.

\subsubsection{Tensions paradigmatiques au sein du legal complex}

A défaut d'entamer une analyse approfondie des conditions de possibilité de la transformation du système juridique ${ }^{2}$ - qui n'est pas strictement requise pour éclairer les pratiques présentées ici - je m'appuierai sur un modèle explicatif post-luhmannien (Cauchie, Kaminski, 2007) qui fournit une réponse opératoire. Sous-système du droit capable d'en assurer l'orientation idéologique (promotion d'une vision hostile, abstraite, négative et atomiste de la protection de la société et de ses normes), la «rationalité pénale moderne 3 » (RPM) peut être décrite, à l'instar de beaucoup d'autres corpus cognitifs, comme constituée d'un noyau dur (les théories de la rétribution et de la dissuasion) et d'éléments périphériques susceptibles de changement qui sont faiblement déstructurants tant qu'ils ne se mutualisent pas. L'un des ferments de déstabilisation de la RPM serait l'émergence périphérique - vers 1950-1960 selon Cauchie et Kaminski - d'une conception aidante de la réhabilitation (transformation de l'emprisonnement, mise à distance de l'expertise médico-légale, valorisation des approches par les contextes de vie), face à une conception plus répressive (théorie du pénitencier) : elle autoriserait des innovations comme celle qui est présentée dans cet article - même si la conception substantialiste de l'innovation chez ces auteurs est très différente de celle qui sera pratiquée ici.

C'est bien à une évolution des conceptions pratiques de l'appareil judiciaire, de la sanction par la séparation à la sanction par la réintégration ("l'état de saisine permanent» - Garapon, 1991 : 224-225), i.e. l'avènement de la justice restauratrice, qu'il faut attribuer les nouvelles possibilités d'agir que manifestent les prises en charge sociales rationalisées de grande ampleur des violences conjugales : elles s'articulent manifestement à la réhabilitation aidante. Des «possibilités d'agir» auxquelles les conceptions restauratrices, les incitations économiques et les transformations de l'Etat-Social (Delage, 2015 ; Bernheim, Commaille, 2012) donneraient latitude. Latitude mais non pas création ex-nihilo: les premiers matériaux pour la construction d'un problème ont été réunis par les féministes (Cardi, Devreux, 2015). Si l'on modifie encore la focale, on peut supposer que la poussée des prises en charge d'urgence (initialement amateures) a conduit l'institution politique à solliciter la mise en mouvement de l'institution judiciaire (Cador, 2005 ; Commaille, 2010). Je traiterai comme un processus de politisation (i.e. un mode de régulation, par des dispositifs gradués, de l'entrée dans la Cité : AUTEUR, c) ce phénomène, constatable dans bien d'autres domaines et qui a fait le succès des différentes formes de médiation.

\subsubsection{Cristallisations proto-juridiques}

\footnotetext{
${ }^{2}$ On peut les réduire à deux dimensions : le rapport au passé et le rapport à l'environnement. Quant à la première, s'agit-il de "dépendance au sentier » (path dependence) - c'est-à-dire du poids invisible des choix antérieurs; de sédimentation; ou de conversion (Thelen, 2003) ? Quant à la seconde : le continent juridique parvient-il à réguler ses rapports avec son environnement par autopoïèse (Luhmann, 1989), c'est-à-dire par la construction de filtres plus ou moins ingénieux pouvant réduire l'événement législatif à du «bruit» (Teubner, 1987) ou au contraire finit-il par subir un « retournement» qui en fait un "produit» (Supiot, 2010:64) ?

3 « Moderne » a le sens que lui donnent les historiens et concerne ici un mouvement émergeant au XVIII ${ }^{\dot{e}}$ siècle.
} 
Mais - et c'est le deuxième moment, d'ordre méso-social - ce territoire politiquement et juridiquement construit et consolidé fait retour sur ses conditions de production et en redéfinit au moins certaines modalités d'application. Pour y parvenir, les animateurs de cet espace de prise en charge ont sans doute pu bénéficier des conquêtes théoriques et pratiques réalisées sur d'autres terrains du social et se sont sentis autorisés à réfléchir à des politiques d'équipement proto-juridique susceptibles de traiter les manques et insuffisances du processus de politisation existant, auxquels ils se heurtent au quotidien. Ces réflexions se sont cristallisées (AUTEUR, c) autour d'un impératif de continuité et de cohérence au sein des prises en charge policière, judiciaire, sanitaire et sociale. Elles ont ouvert la voie à de nouveaux équipements de travail (en général traités comme des "dispositifs»). Ainsi, les organismes de prise en charge de plus en plus professionnalisés (par la formation de leurs intervenants et par leurs outils d'intervention) qui se voient confier/déléguer des missions d'accompagnement para- ou péri-judiciaire (concernant les personnes identifiées comme « auteurs »), de prévention et d'accueil, n'entendent pas assurer seules ces missions. D'une part, elles refusent toute forme de confinement à des experts d'un problème qui interroge l'ensemble du corps social et en premier lieu les instances judiciaires (et policières) et d'autre part elles s'estiment paradoxalement moins bien placées que les professionnels « de première ligne » pour traiter les formes les plus immédiates de ces violences. Moins bien placées parce qu'elles fondent leurs outils d'intervention sur une vision du monde autonomiste (dont les valeurs sont : responsabilisation, proximité, projet) largement répandue aujourd'hui tant dans l'intervention sociale que dans les entreprises (Boltanski et Chiapello en ont fourni une fameuse modélisation) ${ }^{4}$.

\subsubsection{Interpellations ontologiques}

Considérons le troisième moment, lui aussi méso-social où s'opère la synthèse des deux moments précédents : tout l'acquis consolidé pendant la trentaine d'années où ce processus de professionnalisation s'est manifesté (pour la prise en charge des victimes, car pour celle des auteurs c'est plus récent et plus immédiatement professionnalisé) est mobilisé comme politique ontologique ${ }^{5}$ (Mol, 1999) de «dissémination ». Cela signifie que les appareils judiciaire, policier et d'intervention sociale sont interpellés pour passer à un degré supérieur de politisation, afin d'être à la hauteur des performances sociales qu'ils ont suscitées et que cette interpellation acquiert la force d'un impératif. Le judiciaire est invité à ne pas se réfugier derrière l'impersonnalité de décisions relevant du paradigme majeur de la théorie de la réhabilitation (la peine ou le classement), voire derrière quelques initiatives timides relevant du paradigme minoritaire (des transferts limités de compétence du juridique vers le social). On demande simultanément au social de prendre sa part de la tâche de premier secours sans attendre l'arrivée des spécialistes. Et enfin, on attend du monde policier qu'il assume sa mission de sécurisation sans s'abriter derrière une conception restrictive et genrée de la

\footnotetext{
${ }^{4}$ On notera, sans pouvoir le développer davantage, qu'admettre cet effet en retour prémunit contre toute tentative d'application mécanique de la justice restauratrice : on y donne la priorité à la rencontre des différentes « lignes » au détriment de la médiation directe entre les protagonistes de la violence conjugale. Comme on le verra, victimisation et responsabilisation (deux principes « restauratifs») sont travaillés en des lieux soigneusement séparés les uns des autres.

${ }^{5}$ L'expression forgée par Annemarie Mol, qui inverse les rapports usuels entre «ontologie » et «politique », entend insister sur la théorie du réel qu'installe toute initiative politique et, par contrecoup, sur l'absence d'un conditionnement ontologique de l'action politique (c'est l'acte politique qui est ontologique et non l'ontologie qui aurait un prolongement politique). Plus loin, considérant spécifiquement les initiatives ambitieuses de nos intervenants activistes, on parlera de « politique professionnelle ontologique ».
} 
sécurité. Sur le papier, chacune de ces interpellations est prioritaire au même degré, mais on verra qu'il demeure quelque flottement dans leur hiérarchisation plus fine.

Pour s'en approcher, on s'intéresse ici à une version des deux derniers moments (la cristallisation en termes de dé-confinement et la re-politisation attendue) de cette dialectique, sur un terrain restreint (des dispositifs incitatifs de coopération et de formation), en suivant un processus d'innovation sociale en Wallonie. On ne sous-estime pas pour autant l'importance qu'il y aurait à prendre la mesure des arbitrages d'arrière-plan effectués dans ces trois domaines en relation avec l'instance politique.

\subsection{Observer le travail de construction de l'offre et de sa réception}

Le choix d'un terrain d'étude en Belgique, nous oblige à tenir compte des particularités du champ politique (Vigour, 2009) et des cultures juridiques et judiciaires belges - en général considérées comme "mixtes » entre les systèmes accusatoire et inquisitoire (Vigour, 2004). Si le type de relation des procureurs avec l'autorité politique mériterait aussi qu'on s'y attarde, on s'intéressera davantage à leur fonctionnement en tant qu'instance collective de travail, que Milburn et al. (2010: 183-191) décrivent comme orientée par " un team spirit » (op.cit. : 189), qui va bien au-delà de la création du collège des procureurs du roi.

Comme on le verra, ces spécificités sont ici utilisées pour éviter toute transposition mécanique d'une réalité nationale sur une autre, mais sans ambition comparative. Le dispositif wallon observé est considéré comme une innovation, non pas parce qu'il est pour l'instant très peu répandu sous une forme fonctionnelle significative (même au Québec, source de l'expérimentation, il n'a pas cette ampleur), mais parce qu'il modifie la donne : il change l'idéologie de prise en charge, les positions des intervenants et le rôle du dispositif judiciaire. Il nous faut dès lors préciser les outils théoriques et méthodologiques nécessaires à leur analyse.

\subsubsection{Les enjeux : des ontologies professionnelles en situation d'innovation}

L'une des difficultés qu'affronte cet article est de parvenir à se situer par rapport à plusieurs traditions disciplinaires que notre thématique semble imposer. Se limiter à la sociologie ne simplifie pas forcément la tâche : analyser les marges de manœuvre de l'institution judiciaire face aux violences conjugales engage au moins à se confronter à des résultats ou des débats de sociologie du droit, comme on vient de le voir, mais aussi à ceux de la sociologie des politiques publiques (lorsqu'il s'agit des politiques pénales et sociales). S'intéresser aux Parquets, c'est tirer parti de la sociologie des professions juridiques (pour leur cadrage : Milburn et al., op. cit.), tandis que la prise en charge militante des violences conjugales, en lien complexe avec la professionnalisation, nous tire vers la sociologie du travail social et du monde associatif. C'est pourtant la sociologie de l'innovation qui constituera l'axe fédérateur de tous ces apports. Il n'y a là rien d'automatique : il se trouve que la singularité des collectifs de travail étudiés dans cet article tient à leur ambition de construire un dispositif collaboratif insolite pour le traitement, en Belgique, des deux versants des problèmes de violences conjugales : la prise en charge des victimes et celle des auteurs. Pour traiter la revendication d'innovation de ce groupe d' " entre-preneurs » (ils s'assurent des prises en s'interposant), on s'inspirera de la modélisation testée initialement par Michel Callon et largement réajustée depuis (notamment pour résister aux réutilisations figées de la sociologie de la traduction) : on retiendra donc quatre phases qui jalonnent le processus d'innovation (problématisation, intéressement, enrôlement et mobilisation), même si les trajectoires s'avèrent beaucoup moins 
tranchées et irréversibles qu'on ne le croit parfois (Akrich et al., 2006) ${ }^{6}$. En termes plus directs, ce que nous allons analyser dans les sections qui suivent c'est la construction d'une offre et le travail de réception qu'elle entraîne. Les résultats d'ores et déjà empiriquement validés trouvent dans ces éléments de cadrage théorique et dans leur mise en contexte d'innovation, une portée syntagmatique, i.e. relative à la logique du domaine considéré (en gros, la relation du juridique au social) et simultanément thématique, i.e. transposable à des domaines homologues ${ }^{7}$.

Les enseignements sont syntagmatiques si l'on considère les présupposés fondamentaux des interventions spécialisées (des politiques professionnelles ontologiques). Il s'agit alors premièrement d'observer le degré de pénétration d'une idéologie autonomiste de prise en charge des violences conjugales (la dissémination) dans les systèmes judiciaire, policier et social en Wallonie. Deuxièmement, de comprendre comment cette forme de compétence partagée (entre spécialistes et entre spécialistes et non-spécialistes) se distribue dans des pratiques d'intervention qui ont été construites comme étanches. Troisièmement, de mesurer l'utilisation d'une marge de manœuvre parquetière structurelle (le principe d'opportunité) dans une situation déstabilisée, où les dispositions héritées de la formation initiale (surtout celle des magistrats et des policiers) sont en décalage avec l'évolution de la situation des prises en charge et la relative immobilité des dispositifs juridiques, même si ce décalage n'est pas uniforme comme le notaient déjà, pour les magistrats, Faugeron et Jakubowicz (1984).

Sur le plan thématique (ou si l'on veut des analyses substituables), l'imposition d'une grille de lecture en termes de sociologie de l'innovation nous confrontera à des enjeux plus transversaux, rattachant le propos à une anthropologie de la connaissance dans les organisations. Il s'agit notamment des ambiguités profitables qui apparaissent dans les dispositifs d'intéressement à l'innovation : malentendus fonctionnels à propos des rôles à tenir et conceptualisations rassemblant plus par leurs connotations que par leur dénotation au point d'être objets-frontières. Il s'agit aussi de la pluralité des attachements des intervenants aux équipements mis en œuvre - une pluralité que les innovateurs considérés ici n'ont pas forcément théorisée mais dont ils tiennent fortement compte pour avancer sans attendre l'adhésion uniforme à leurs problématisations.

C'est, on l'espère, l'assemblage de ces résultats syntagmatiques et thématiques « immédiats » qui pourrait donner quelques pistes de réponses à plus long terme aux problèmes soulevés par la dialectique globale du juridique et du social qui a été exposée dans le point précédent.

\subsubsection{Matériaux et méthodologies}

Le matériau exploité pour cet article est tiré d'une observation participante de quatre ans (2008-2012) au sein de trois associations sans but lucratif (a.s.b.1.) en Région wallonne. Deux d'entre elles prennent en charge, sous différentes formes, les victimes (le « Collectif contre les Violences Conjugales et l'Exclusion » à Liège ; "Solidarité-Femmes » à La Louvière) et l'autre travaille avec les auteurs («Praxis», à Liège et à La Louvière) ${ }^{8}$. Sur l'aire

\footnotetext{
${ }^{6} \mathrm{Ce}$ modèle s'applique bien à des innovations par ajout (un nouveau dispositif). Il devrait être nuancé pour des « innovations par retrait» (Goulet, Vinck, 2012) comme pourraient l'être des dispositifs de circuits courts intrajudiciaires, telle la médiation pénale ou les Maisons de justice.

${ }^{7} \mathrm{Je}$ reprends ici la distinction syntagmatique/thématique formulée par Jean Cavaillès pour la théorie de la science (Cavaillès, $1960: 27-30)$.

${ }^{8}$ L'a.s.b.l. «Collectif contre les Violences Conjugales et l'Exclusion» (CVFE), compte une soixantaine de salariés œuvrant à l'accueil (gestion d'un refuge) et à l'information auprès des victimes ; l'a.s.b.l. « Praxis » a une quinzaine de salariés, travaillant - aux trois quarts sous mandat judiciaire - auprès des auteurs. À La Louvière, l'a.s.b.l. « Solidarité-Femmes » est une association d'une trentaine de salariés aux missions voisines de celles du CVFE. Leurs noms apparaîtront désormais sans guillemets.
} 
géographique de référence, ces trois associations sont les principales interlocutrices des autorités publiques parce qu'elles sont à peu près les seules à proposer une prise en charge globale des violences conjugales - les autres a.s.b.l. ayant des objectifs plus circonscrits (information, conseil). Ces associations ont souhaité disposer d'un regard extérieur sur l'expérience de travail en commun tout à fait inusitée qu'elles venaient de commencer avec le soutien de la Région wallonne : c'est ce qui a fourni l'occasion d'un suivi de terrain (une mission dont les fondements sont analysés ci-dessous en 2.1.1.). Je n'utiliserai - et à des fins uniquement illustratives - qu'une faible partie des données récoltées : les 123 heures de suivi du travail des équipes en situation de coopération, les 154 heures de participation au comité de pilotage avec les Directions ont été mises à contribution dans le cadre d'un autre article (AUTEUR, d), même si c'est ce matériau qui m'a permis de comprendre celui, plus restreint, que je mobilise ici. J'aurai donc principalement recours à des documents écrits et à des entretiens semi-directifs: trois bilans annuels (les Rapports de 2009 et 2010 de ces associations à la Région Wallonne) et 25 entretiens de portée générale avec des professionnels de première ligne (huit avec magistrats, policiers, Services d'Aide aux Justiciables - SASJ et 17 autres avec d'anciens participants aux formations). Les caractéristiques socioprofessionnelles de ces personnes seront précisées lorsque leurs réponses seront utilisées une utilisation qui tiendra compte du fait que la représentativité de cet échantillon ne porte pas sur l'ensemble des milieux professionnels concernés, mais plutôt sur cette frange qui est volens nolens en contact avec les problématiques de violences conjugales.

Comme cela a été le cas pour le matériau d'observation, les sources documentaires et les entretiens ont été traités à partir d'une grille d'analyse thématique, construite sur la base de la problématique qui a été esquissée ci-dessus, mais réajustée à plusieurs reprises au cours de ce long processus.

\section{Théorie et pratique de la coopération en situation de méfiance}

La problématisation macrosociale que j'ai proposée est applicable à des situations de prise en charge des violences conjugales dans différents pays européens, sans doute au prix de quelques ajustements. Elle ne suffit pas à faire saisir les relations complexes de la politique, du droit et de l'intervention sociale : ce qui apparait en tout cas, c'est que lorsque le modèle usuel de traitement de ces conflits domestiques est posé comme insuffisant (ici : trop clivé), des problématisations issues du terrain associatif émergent et, sans être directement productrices de droit - les juristes associatifs pratiquent en général plutôt une expertise d'attribution («Elles ont des juristes. Je reçois des courriers parfaits »: Magistrat, Liège) elles ouvrent de nouveaux espaces d'action institutionnelle et dans certains cas sont indirectement à l'origine d'évolution législative.

En quoi cette problématisation émergente (premier acte d'une innovation) se singularise-telle ? Très synthétiquement, on peut la ramener à la conceptualisation explicite de trois pratiques de terrain : 1 / une conception systémique du conflit domestique, 2 / une réévaluation de la pertinence des interventions spécialisées et $3 /$ une refonte des rapports entre intervention spécialisée et intervention de premier contact. On notera qu'aucun de ces outils n'est véritablement original, mais lorsque ces «entre-preneurs » transportent de manière résolue ces instruments sur un terrain où ils ne l'étaient pas, ils transforment la bonne idée en innovation. La première conceptualisation, pleinement théorique sera examinée d'abord (2.1.), les deux autres, qui participent d'une réflexion beaucoup plus ancrée seront exposées dans un second temps (2.2.).

\subsection{Une conception systémique des violences conjugales}


Les intervenants sociaux sur les violences conjugales ne partent pas de rien : au fil des ans, ces interventions, d'abord inspirées par une philosophie et une politique de la libération des femmes ont pu trouver des points d'appui sérieux dans les travaux des psychologues et des criminologues et mettre sur pied un cadre d'analyse qui donne à leur militance une stabilité dans la durée. Mais les innovateurs que nous suivons étaient depuis longtemps insatisfaits de ces routines de travail, qui pouvaient ainsi s'autoriser des conceptualisations académiques existantes. Soucieux de remonter du fléau qu'ils traitent à ses multiples racines, ils sont allés puiser à d'autres sources leur permettant de globaliser leur analyse.

\subsubsection{Un cadre d'analyse peu opératoire}

Quelle est la situation initiale ? Le cadre général des prises charge dans la plupart des pays développés, est celui de la séparation des interventions (Herman, 2011, 2012). D'un côté, des personnes entrent dans la zone d'action des associations accueillant des victimes et sans sortir de cette zone (parfois physiquement même, sans sortir des lieux dévolus) commencent leur travail de victimisation / dé-victimisation et de réhabilitation; de l'autre, des personnes, le plus souvent sous injonction judiciaire, mais pas exclusivement, intègrent d'autres types d'associations où elles sont incitées à se responsabiliser comme auteurs de violences domestiques. Non seulement ces interventions sont séparées, mais elles entretiennent en général peu de rapports sinon de méfiance. C'est pourquoi on peut se servir de la puissance évocatrice d'une métaphore proustienne : certaines choses se font «du côté de chez » les auteurs, qui ne se font pas « du côté de chez » les victimes et inversement.

Cette séparation des mondes obéit à des raisons à la fois situationnelles (la charge émotionnelle des conflits est telle qu'elle semble impliquer l'éloignement des protagonistes) et historiques (la prise en charge des personnes subissant les violences est née dans la nébuleuse féministe qui a longtemps nourri des doutes sur les motivations profondes de la prise en charge des auteurs, d'où l'absence de relations). Même si le processus de régulation politique et juridique des interventions sur cette base présente des spécificités dues à la structure fédérale de l'Etat (Walque, 2008) et aux effets de la " pilarisation " ", il pourrait être largement transposable à d'autres Etats européens.

Les insuffisances de ce système d'intervention clivé ont souvent été pointées, parce qu'il s'affadit en une utilisation banalisée de la notion de "cycle de la violence conjugale » et en une lecture étroitement psychosociale de ces conflits (Cador, 2005). Quant aux limites des dispositifs préventifs ou pénologiques de substitution, ce sont celles qui affectent la pertinence des médiations (Cresson, 2002 ; Faget, 2004), le contexte belge n'apportant là encore aucune différence significative (Cartuyvels, 2004 ; Dubois, 2008), sinon l'incidence de la variabilité régionale ou zonale (Vanneste, 2016). Ce qui frappe dans le cas étudié ici, c'est la convergence des efforts pour problématiser d'une manière singulière les outils d'analyse et les pratiques.

\subsubsection{Outils systémiques pour analyser le processus de domination conjugale}

Les activistes que nous suivons ont une analyse critique de la lecture psychologisante des violences domestiques : "on se heurte à une vision familialiste des problèmes de la violence conjugale. On se heurte à des intervenants qui se limitent à la sphère du couple» (remarque

\footnotetext{
${ }^{9}$ En Belgique, on estime qu'une très grande partie des initiatives d'intérêt public repose sur trois piliers principaux qui combinent les influences chrétienne et laïque et qui doivent être en équilibre : les piliers libéral, socialiste et social-chrétien. L'impact de la pilarisation (verzuiling) sur les politiques publiques, difficile à mesurer dans le détail, est en général considéré comme important. Quoiqu'il en soit, évaluer la contribution de ce filtrage à la fabrication des dispositifs dépasserait le cadre de cet article.
} 
d'une Directrice, Côté-victimes, au conseiller du ministre, à Jambes le 16/12/08). C'est l'analyse systémique qui leur a servi de trame pour favoriser l'implantation d'outils conceptuels nouveaux sur le terrain des violences conjugales, sans doute parce que certains de ces professionnels étaient sensibilisés à cette grille de lecture, qui offre un pont commode entre l'interprétation psychologique et l'interprétation sociologique de ces phénomènes (comme le montre le cas des thérapies familiales). La familiarisation avec des idées comme le traitement de la pathologie par le contexte, le principe d'interdépendance des actes et plus globalement l'importance d'une vision en termes de complexité, ont pu jouer un rôle important. Certes, l'application des principes systémiques à de nombreux domaines de l'action sociale est bien connue et fait partie de l'ordinaire des programmes de formation dans les écoles de travail social et dans les facultés de psychologie. Mais il y a loin de la connaissance d'une philosophie des rapports sociaux - et même de son utilisation en pratique comme méthode de traitement de cas - à la construction rigoureuse d'un protocole de travail pouvant s'appliquer simultanément à tous ceux qui sont concernés par la violence conjugale et intrafamiliale.

Pour les membres des associations considérées ici, le facteur de réduction de cette distance a été la mise au point, au Québec, d'un outil de travail performant (Ayotte et al., 2007). Les dirigeants d'un foyer d'accueil pour femmes victimes de violences conjugales (la Séjournelle), ceux d'un centre de prise en charge d'hommes auteurs de ce même type de violences (l'Accord Mauritie) et des psychologues de l'Université du Québec à Trois-Rivières ont conçu un dispositif destiné à associer ceux qui sont dans la prise en charge "du côté de chez les victimes" et ceux qui sont "du côté de chez les auteurs": le Processus de Domination Conjugale (l'appellation couramment utilisée sur le terrain étudié est « PDC »). Il s'agit d'une grille d'analyse qui affine le «cycle de la violence conjugale » en élargissant le contexte requis pour l'explication et en coordonnant les interventions des différentes parties prenantes de la prise en charge (aussi bien les premiers que les seconds rôles). Son objectif majeur (assurer la sécurité des victimes d'abord, mais aussi des autres membres des familles) reste conforme à la philosophie d'ensemble des organisations s'occupant des effets des violences domestiques en Europe.

Le PDC s'en distingue cependant par plusieurs traits. Il met l'accent sur le repérage gradué des situations dangereuses au sein de la famille (conceptualisés en termes de degrés de sévérité et de dangerosité), d'une part en prêtant attention aux mécanismes facilitateurs (stratégie de contrôle, positionnement de protection, co-apprentissage de la domination et de la victimisation, importance des processus de justification), dans lesquels ni l'auteur, ni la victime, ni leur environnement immédiat ne sont considérés comme passifs et d'autre part en recueillant des données sur la socialisation des deux groupe familiaux initiaux comme du couple lui-même. Le PDC établit en outre que l'intervention conjointe des deux côtés (dans son vocabulaire : la coopération intersectorielle) est la condition de sa mise en auvre optimale. Cela le singularise également: cette condition serait difficile à remplir dans de nombreux pays. On notera enfin une autre originalité de problématisation: bien que ses promoteurs soient parfaitement avertis de l'existence de violences conjugales dont les auteurs sont des femmes et soient prêts à des prises en charge adaptées, "les termes dominant, dominée et victime sont sexués puisque le modèle du PDC a été élaboré à partir de l'observation terrain de victimes féminines et de dominants masculins » (Ayotte et al., 2007 : 2). Ce qui signifie notamment, que le modèle peut rester sexué de cette manière tant que les représentations de la relation de couple continuent d'obéir au schéma de la domination masculine (on pourrait dire, dès lors, que les femmes qui violentent leur conjoint extériorisent 
la domination masculine dont elles ont intériorisé la fatalité $)^{10}$. Une autre caractéristique distinctive de ce modèle (la théorisation des impuissances) sera reprise ci-dessous.

\subsubsection{Eclairages sociologiques et pénombre juridique}

Le recours à la sociologie est la troisième caractéristique de cette partie théorique du travail de problématisation. Il singularise encore plus le groupe belge (lequel travaille néanmoins en étroite relation avec les auteurs du modèle, qui en ont le copyright), car la source majeure de réflexion de ses inspirateurs québécois est la psychologie. Je ne prétends pas que la sociologie reconfigure les outils conceptuels et d'intervention ou devient un bien commun pour les salariés de ces associations : on en est assez loin. Peut-être même la décision de confier le suivi d'action à un sociologue - en l'occurrence l'auteur de cet article - a-t-elle quelque chose de circonstanciel, comme semble l'indiquer la présentation de sa mission, qui insiste sur l'aspect scientifique plutôt que disciplinaire: "A l'initiative du Comité de pilotage, l'accompagnement du projet a été confié à X., professeur de sociologie (...) qui apporte son éclairage scientifique sur la question de la coopération en situation de violence conjugale. Il relève les aspects saillants des processus de coopération (...). » (Rapport d'activités de 2008 : 11 - Région wallonne ; les caractères en gras sont dans le texte).

Toujours est-il que cette décision a donné une coloration spécifique à l'usage de la systémique et à l'analyse des socialisations familiales, en raison, à la fois, de la longueur du suivi (près de quatre ans), des discussions au sein des "comités de pilotage » et des exposés faits par le sociologue aux directions (sur le travail inter-équipes, sur la sociologie de la justification) et aux équipes (sur la sécurité, sur l'empowerment et sur la domination) ${ }^{11}$. Sans doute faut-il ajouter à cette dimension circonstancielle et volontariste, l'effet indirect des caractéristiques du personnel impliqué dans ces prises en charge : à côté des salariés sans spécialisation professionnelle initiale, des psychologues et des assistants sociaux, on trouve notamment des criminologues employés comme tels (ce qui fait une grande différence avec la France où la formation existe, mais les emplois très peu). Par leur formation, ils sont plus réceptifs à des problématiques incluant des socialisations et des milieux de vie structurants.

On ne manquera pas de noter que les débats théoriques des juristes ne participent guère à l'élaboration de cette problématisation. Et pourtant certains des concepts qui sont au cœur de la réflexion de ces organisations sont par ailleurs au premier plan des préoccupations de nombreux politiciens, des juristes et criminologues : ainsi en va-t-il de la sécurité, de la dangerosité ou de la victimation ${ }^{12}$. Soit par exemple le mot commun « dangerosité » : le débat juridique et criminologique sur l'internement et la mise à disposition du gouvernement (Mary et al. 2007) est très peu transposé dans les sphères que nous étudions et inversement. Et pourtant il semble très proche (l'une de ses traductions, le " code couleurs » dans le PDC cinq degrés de dangerosité allant du blanc au rouge - pourrait être un outil d'appréciation pour le juge et pour le policier). Tout se passe comme si les activistes estimaient possible de tracer leur route sans attendre, en utilisant les contradictions de la RPM décrites par Cauchie et Kaminski (op. cit.), alors qu'on pourrait, sur le papier, considérer que ces objectifs

\footnotetext{
${ }^{10}$ Ce qui s'entend toutes choses égales d'ailleurs, car dès que la définition des violences conjugales devient plus stricte, la part des auteurs femmes diminue, comme le montrent des données récentes en Belgique (Vanneste, 2016 , résumé : 2).

${ }^{11}$ Il faut évidemment distinguer la sociologie systémique qui inspire ces intervenants sociaux (on s'efforce de la présenter ici dans sa fonctionnalité analytique) et la sociologie pragmatique utilisée par moi pour situer cet outil d'analyse dans un ensemble de pratiques. Le fait que je ne sois pas systémicien n'a à aucun moment posé problème à ces militants.

${ }^{12}$ Lorsqu'il s'agit du processus de retour sur soi comme victime, les associations parlent de « victimisation » (un anglicisme, malgré les apparences) et je les suis. Je réserve "victimation» (un néologisme conforme au français) pour désigner une partie des statistiques pénales.
} 
sociétaux (lutter contre les violences conjugales) ne pourraient être atteints qu'au prix d'une rationalité pénale restructurée, qui à défaut d'être "post-moderne», serait celle de la «modernité radicale » conceptualisée par A. Giddens et U. Beck. C'est plutôt par cette problématisation à l'état pratique, complément indispensable des fondements théoriques qu'on vient d'examiner, que les innovateurs rejoignent juristes, politiciens et criminologues, non point dans les enceintes académiques, mais in the wild.

\subsection{Un travail sur les lignes : coopération et dissémination}

Comme on le voit, la dénonciation des insuffisances de l'appareil juridique ou des modalités de sa traduction judiciaire ne sont pas au premier plan, même si, comme on l'envisagera tout d'abord, l'impulsion donnée par des chefs de parquet emblématiques a été décisive : tout se passe comme si ces agitateurs concevaient leur action comme possible en utilisant l'existant (normes, textes, jurisprudence), sans s'interdire de mener, sur d'autres plans, un travail à long terme de persuasion politique pour modifier au moins les textes et les pratiques judiciaires. On l'a suggéré dès l'entame de cet article : ces innovateurs demandent bien sûr aux magistrats et policiers d'être plus systématiques à l'égard des auteurs (que l'acte reçoive une réponse, même insuffisante, plutôt que le message socialement paradoxal du classement sans suite), mais ils veulent aussi qu'ils soient plus attentifs à la gravité différentielle des situations (que l'on déploie toute la palette de mesures disponibles plutôt que la poursuite sèche et que l'on soit aussi soucieux de la prévention). Sur cette base, les militants installent un discours inattendu : densifier le réseau des interventions de première ligne plutôt que raffiner le geste spécialisé, donner toute sa place à l'expérience qui y est vécue.

\subsubsection{Incitations politiques, volontarisme judiciaire et audace associative}

Le travail de problématisation est largement alimenté par l'incessante inventivité pratique de ces groupes lorsqu'il s'agit de tirer parti du contexte politique. La base politico-juridique de leurs dispositifs d'intervention s'est affirmée, pour la Belgique, en avril 2006, avec les Circulaires communes de la Ministre de la Justice et du Collège des Procureurs Généraux définissant les violences au sein du couple («COL3») et donnant des orientations aux Procureurs du Roi et aux forces de l'ordre en matière de violences entre partenaires ( COL4»). Mais la mise en route du dispositif wallon a bénéficié en outre d'initiatives politiques et judiciaires suffisamment originales pour être désormais régulièrement évoquées, par les militants de l'intérieur (Bégon, 2007) et de l'extérieur (Pêcheux, 2012), comme par les chercheurs (Born, Glowacz, 2006), en tant que premières bornes du parcours de légitimation de la lutte contre les violences conjugales en Belgique. À partir de 2004, dans la Province de Liège, ce seraient : un député provincial sensible à la concertation socio-judiciaire (ce n'est pas le cas partout, encore aujourd'hui, me disait un magistrat), au Parquet de Liège une procureure à l'initiative d'une politique de «Tolérance zéro » (c'est-à-dire : le refus du classement sans suite) sur les violences conjugales, le tout se matérialisant dans la mise en place d'une « Coordination provinciale » des principaux professionnels concernés.

Encore fallait-il que ces initiatives généreuses trouvent un écho au sein des principaux intervenants de terrain et notamment des deux "côtés ", clairement sollicités de s'entendre alors qu'ils s'étaient construits sur le mode de la séparation. Ce fut le cas en 2006-2007 à Liège, avec l'introduction auprès de la Région wallonne (R.W. désormais) d'un dossier de soutien pour un dispositif de travail en commun des a.s.b.l. Praxis et CVFE. : les "Pôles de ressources spécialisées en violences conjugales et intrafamiliales » (familièrement désignés comme « les Pôles »), organisés selon trois axes de développement : le travail sectoriel auprès des victimes, des auteurs et des enfants; le travail intersectoriel réunissant les équipes hors 
des situations d'intervention; la formation des partenaires. Sans insister exagérément sur l'impact des personnalités facilitatrices, il faut relever que ce projet a été au départ porté à bout de bras par les directions de ces deux organisations, qui ont fait le pari du dépassement de leurs cultures de travail. Ce dossier élargi au Hainaut (collaboration entre Praxis et Solidarité-Femmes à La Louvière) aboutit en fin 2006. Si les ressorts profonds de cette extension restent hors du champ de cet article, il faut tout de même souligner que l'appui de la R.W. est à la fois fort (une affectation à ce seul projet, pendant plusieurs années, de tout le budget fléché) et ambitieux (l'attente d'un travail de modélisation).

Selon ses promoteurs, un tel rapprochement pourrait avoir d'importantes répercussions indirectes sur la qualité de la prise en charge pour toutes les parties prenantes, c'est-à-dire pour les victimes, auteurs, enfants ("Le fait de penser à la ressource qu'offre le servicepartenaire pour le traitement d'une situation contribue à offrir une meilleure approche de celle-ci» (Rapport de 2009, R.W.) et permettrait d'affiner le travail aussi bien des intervenants spécialisés que des services auxquels victimes et auteurs ont recours. Mais comment inscrire cet objectif dans la réalité vécue par les équipes?

\subsubsection{Cultures professionnelles et revendication d'expertise}

Le premier élément de cette problématisation organisationnelle en cours d'action (souvent effectuée sur le mode de la réflexivité en collectif) relie la réussite d'un processus professionnel explicitement posé comme innovant au respect des identités initiales et des temporalités d'intervention propres à chaque "côté ": la coopération vient " $s$ 'inscrire dans des cultures professionnelles respectives existantes », c'est pourquoi «chaque intervenant n'est pas contraint d'y arriver au même rythme mais c'est une perspective vers laquelle les associations constitutives des Pôles ont décidé de tendre. Il s'agit d'un choix institutionnel, orienté vers l'innovation sociale, permettant d'élargir l'approche de la problématique complexe des violences conjugales et intrafamiliales » (idem - les caractères gras sont dans le texte). La coopération suppose reconnaissance mutuelle, partage, protocoles, régularité, mais ne gomme pas les conflits: "Accepter de faire évoluer ses représentations passe par la confrontation, parfois même par l'affrontement » (idem). Ces conflits sont alimentés par des représentations solidement ancrées du public auprès duquel on intervient : en l'occurrence une difficulté "pour des services d'aide aux victimes, de concevoir qu'un auteur des violences est, au même titre qu'une victime, capable de modifier ses comportements » et "pour un service d'aide aux auteurs, de tenir compte de la victime et des enfants » (idem). Par contrecoup, il est difficile de "faire bouger la représentation qu'on se fait du métier de l'intervenant "d'en face" "(idem). De fait, après une année d'euphorie, les tensions entre "les deux côtés" au sein des Pôles ont été particulièrement vives, à Liège comme à La Louvière, entre septembre 2008 et mars 2009. Cette coopération modulaire est un défi pour les organisations dont les directions sont incitatrices alors qu'une partie des équipes est sur la

réserve. Sur ce point également, la problématisation bénéficie de la réflexivité, comme le montre cette remarque sur les limites du volontarisme en matière de coopération intersectorielle: "Travailler entre secteurs différents est tellement difficile qu'on ne s'y coltine que s'il y a une nécessité. Cette nécessité, elle s'impose, elle se vit, elle ne se décrète pas» (idem).

Dès lors que des progrès sont enregistrés sur ce chemin, c'est la place du professionnalisme qui se redéfinit : comme on l'a montré ailleurs (AUTEUR, d) le professionnalisme est une question sensible dans ce champ où les premières initiatives, à peu partout en Europe, ont démarré sur un mode plutôt militant; parvenir à le confirmer tout en le décalant nous fait en quelque sorte sortir du débat par le haut (modéliser pour affirmer une expertise). C'est ce que dit clairement le rapport de 2009 - de loin le plus élaboré : «L'installation et la consolidation 
des Pôles démontre l'existence d'un professionnalisme (articulation de savoirs et de dispositifs d'utilisation). Ce professionnalisme ne va pas cesser (notamment parce que les porteurs de l'action demeurent, partiellement du moins, eux-aussi des acteurs de première ligne) (...) mais il doit donner naissance à une nouvelle dimension : l'expertise (la capacité d'intervenir sur des situations que le seul regard professionnel ne peut pas dénouer) ; qui plus est, cette expertise a une dimension beaucoup plus participative (et incitative) qu'exclusive; qui plus est, cette expertise consiste à susciter des réseaux et à y participer, donc sur le mode wiki. Plus on avance vers les non-spécialistes, plus le travail de modélisation devient nécessaire et plus le travail des modélisateurs devient une expertise » (idem).

\subsubsection{Un dispositif d'essaimage modifiable par ses destinataires}

La prudence de cette conception processuelle et différentielle de la coopération entre équipes spécialisées devient, comme on peut le lire ci-dessus, ouverture lorsqu'il s'agit des relations avec tous ceux qui sont confrontés, sans y être nécessairement préparés, à l'engrenage des violences conjugales - ceux qu'on dit être «en première ligne ». C'est la seconde dimension de la problématisation organisationnelle : rechercher la justesse des gestes accomplis par les professionnels qui seront les premiers touchés (magistrats, policiers, travailleurs sociaux), non pas en leur inculquant des recettes d'intervention, mais en les incitant à transposer, à leur niveau d'action et dans les limites de leur pouvoir d'agir, les outils d'analyse que condense le PDC. La métaphore de la dissémination sténographie cette ouverture vers les «premières lignes »: "Pour s'assurer la conquête de nouveaux milieux de vie, les végétaux disséminent graines ou spores. C'est-à-dire que la fleur laisse accessible au vent, aux oiseaux, aux insectes, ... ses graines pour qu'elles soient transportées au-delà de son espace habituel de reproduction" (idem). Il y a bien des schémas d'action d'experts à transmettre aux professionnels, mais quelque chose des difficultés rencontrées par les professionnels, y compris dans la mise en œuvre fidèle des bons outils, doit revenir bottom up aux experts et les obliger à ré-agencer leurs modèles ("Ce qui est en jeu ici c'est le principe d'un dispositif d'essaimage ouvert et modifiable "par le bas", dans des conditions qu'il faut définir "idem). C'est dire que coopération intersectorielle et dissémination des savoir-faire dans l'environnement sont incompatibles avec la protection frileuse d'un territoire professionnel : "On parle souvent de la nécessité d'“ouvrir les boîtes noires”, pour désigner l'impératif de dévoilement des procédures qui ont permis d'obtenir un produit ou un service. Pourtant la métaphore pourrait-être plus riche encore : la boîte noire enregistre les opérations effectuées avec leurs aléas. La tendance des institutions est plutôt de chercher à protéger leurs savoirfaire et à ne pas donner de détails sur les modalités de production du bien qu'elles proposent (comme si celui-ci avait été obtenu sans aléas). Une telle attitude serait ici contreproductive: l'intervention sur les violences conjugales, à quelque "ligne" qu'on la situe, n'est pas l'application ne varietur d'un schéma tout prêt. Ouvrir la boîte noire des Pôles, c'est donc montrer toutes les difficultés rencontrées, même entre collectifs bien disposés les uns à l'égard des autres » (idem). Comme on va le voir plus loin, la formation est pour l'heure l'équipement privilégié d'une première étape de la dissémination parce qu'elle offre un outil solide (le PDC), un exemple de collaboration des deux côtés (des binômes de formateurs) et des conseils pratiques pour faire face au sentiment d'impuissance (s'en sortir par la recherche de collaborations et de réseaux plutôt que par un surcrôt de savoir; sur ce point : AUTEUR, d).

Si potentiellement les professionnels de première ligne (mais aussi des amateurs) sont nombreux à être concernés par ce programme, tous ne peuvent être touchés à court terme. Comme en convinrent les membres d'un petit groupe de réflexion, réunis à Liège en 2011, il 
faut donc repérer des "conditions favorables» (à Liège, l'impact du passage d'une procureure emblématique, à Mons un substitut spécialisé sensibilisé après une formation et de nombreux intervenants du système sécuritaire formés au PDC) et, en raisonnant par arrondissements judiciaires ${ }^{13}$, "prioriser » l'intéressement puis l'enrôlement du Parquet (droit commun et famille), de la police - les agents de terrain et le Service d'Aide Policière aux Victimes (SAPV) - et, si possible, celui des juges, des Maisons de Justice, des Services d'Aide Sociale aux Justiciables (SASJ) et des instances collaboratives (Plates-Formes et Conseils d'Arrondissement $)^{14}$. Mais là encore «le diable est dans les détails », comme le montre cet échange (où $\mathrm{B}$ répond directement à $\mathrm{A}$ ) :

- "A (côté auteurs, Liège) : J'y crois pas aux policiers, tels que je les connais. On sait que c'est quelque chose de très éphémère, lié aux stat's. Je crois davantage aux procureurs, qui eux pourraient avoir un impact sur la police.

- B (côté victimes, Liège) : Je me dis qu'il y a des chefs de zone de police avec lesquels on peut faire quelque chose. Après... qui tu vises dans la police? Un parquet qui n'a pas son outil, la police, qu'est-ce qu'il peut faire?

- C (côté victimes, La Louvière) : Dans le Conseil d'Arrondissement, le Parquet est là.

On pourrait commencer par une sous-commission. On n'aurait pas intérêt à faire tout ça en dehors [du Conseil].

- D (côté auteurs, Liège) : J'ai des contacts avec des policiers de $2^{e ̀}$ ligne. Ce sont eux et non les agents de crise qui peuvent s'intéresser.

- E (côté victimes, Liège) : Ce sont [pourtant] eux [les agents de $1^{\text {è ligne] qui peuvent }}$ ressentir de l'impuissance.

- C : On n'est pas obligés de les former au PDC. [Mais] ceux qui sont confrontés à la crise sont quand même parfois frustrés de n'avoir pas d'outils.

- D: Il y a eu une séquence hallucinante, avec deux commissaires qui avaient le discours basique (c'est la victime qui provoque)!

- F (co-auteure du PDC, Shawinigan, Québec) : On a eu ça aussi. On leur a dit : pensez ce que vous voulez, mais qu'est-ce que vous pouvez faire pour la sécurité ? Et il faisait!

$C$ 'est l'action (...) On a pu avancer parce que son chef lui a dit qu'il avait une mission de protection (...)» (extrait d'observation d'une séance de brain storming, Liège,

CVFE, 16/11/2011 - je n'ai coupé qu'une partie de la dernière intervention).

Définir les priorités pour ces premiers moments de dissémination ce serait se demander : «À qui fait-on appel quand notre intervention ne paraît pas suffisante? (B) ». Mais les habitudes de travail du côté victimes («Le SASJ est un incontournable, ils font partie du système (...) (B) ») ne sont pas celles du côté des auteurs («Oui, mais ce n'est pas le nôtre. Nous les appels à d'autres services qui nous aident le plus, ce sont les psychiatres, SOS Enfants (...) (A) ». À l'intéressement en cours d'action (cf. : "C'est l'action! ») du plus grand nombre de partenaires peuvent donc logiquement succéder des enrôlements différenciés : "On n'est pas toujours obligés de travailler en intersectoriel... Qu'est-ce qui fait qu'on pourrait avoir recours à des partenaires différents ? (C)»

Sachant que «le partenaire idéal, il est pas toujours mûr pour travailler (F)» (idem), ces militants apprennent au jour le jour qu'ils doivent à la fois maintenir leur intention programmatique rationnelle (la dissémination par la mise en commun des impuissances) et

\footnotetext{
${ }^{13}$ Cette démarche de proche en proche a été validée après coup par le constat chiffré de la grande variabilité des pratiques judiciaires et policières selon les arrondissements (Vanneste, op. cit.).

${ }^{14}$ En région flamande (à Anvers pour l'instant) un « Family Justice Center » vient d'être créé en juin 2016 pour permettre « une approche globale des violences conjugales» par la centralisation locale de tous les services concernés : $\quad$ http://fr.metrotime.be/2016/06/28/news/le-ministre-flamand-jo-vandeurzen-veut-une-approcheglobale-des-violences-familiales/
} 
accepter la mise en œuvre imparfaite de leur modèle: peut-être même est-ce par ces approximations que le dispositif atteint au mieux ses objectifs (AUTEUR, b).

\subsection{Vers une redynamisation de la société civile?}

Suivre le parcours des innovateurs devrait non seulement montrer l'émergence de nouveaux espaces d'interaction droit / société mais aussi identifier, mieux que ne le permettait la modélisation macro-sociale, ce que des porte-paroles du « système des besoins » attendent de «l'appareil juridique (Rechtspflege) 》 et de «l'appareil administratif et répressif 》 - c'est-àdire les composantes de la société civile selon Hegel - et d'entrevoir ce qu'ils construisent avec les autorités politiques (Hegel, 2013, §§ 205, 208, 232, 261) ${ }^{15}$.

«Attendre » et «construire »: ces deux verbes ont de l'importance pour notre propos. La problématisation experte des violences conjugales s'adresse au système judiciaire et au système policier afin de les intéresser (pour qu'ils y trouvent leur intérêt), puis de les enrôler (pour qu'ils jouent un rôle) et finalement de les mobiliser (pour qu'ils soient eux-mêmes des porte-paroles en actes) : les innovateurs n'attendent pas que le droit évolue dans son corpus mais dans sa pratique ; ils n'attendent pas que les policiers deviennent les hérauts de la lutte contre les violences intrafamiliales, mais qu'ils étendent le champ de leur mission de protection. Sur un autre plan (qui peut être d'interpellation ou de pression politiques), en tant qu'ils sont aussi des «collectivités » (Die Korporation) exprimant une éthique professionnelle (id. § 255), ces experts engagent avec la société politique - concrètement ici : la Région wallonne - un travail de construction de nouveaux cadres (id., § 249) de protection et de promotion. Reste quand même en arrière-plan, non résolue, la question des limites procédurales : comment leur volonté politique d'extension du domaine de la sécurisation peut-elle s'ajuster à la contrainte parquetière (attente de l'infraction) ? On y reviendra.

La problématisation macrosociale (savante) du chercheur conduisait à l'hypothèse d'une temporalité ralentie, au sein de la dialectique du droit et du social. Parce qu'elle prend en compte cette durée alanguie, la problématisation méso-sociale des experts les conduit à privilégier l'implication de l'existant (magistrats, police, services sociaux), dans la perspective d'une (re)dynamisation plutôt que de reproduction des routines de la société civile, ce «système de moralité perdue dans ses extrêmes» (id., § 184) : il s'agit pour eux de tirer parti des différences et des tensions pour les convertir en potentialités. Au cœur de l'agon, ils veulent restaurer une agency. La formation est alors l'outil privilégié d'accès à ces nouvelles dynamiques de travail.

\section{Les ambitions de l'enrôlement et les difficultés de la mobilisation}

S'il ne s'agissait que de vérifier l'écho rencontré par une offre d'équipements d'action, l'affaire serait simple et la réponse presque connue d'avance : le tissu social concerné par un traitement des violences conjugales prend ce qui lui paraît acceptable, approprié à sa situation. Donc : la réception n'est sans doute pas à la hauteur de l'offre. Mais ici, on analyse un dispositif d'innovation et ce qu'il s'agit de repérer c'est le degré d'intéressement, l'endossement des rôles, les potentialités de relais (donc de mobilisation de publics plus larges).

L'intéressement du premier cercle de partenaires occupant une position incontournable (des magistrats de référence, travailleurs sociaux de la police, services d'aide aux victimes ou au justiciables) semble acquis. Le défi qu'imposent aux innovateurs les partenaires qu'ils

\footnotetext{
${ }^{15}$ Le recours à Hegel (on le trahit un peu en le dés-historisant) sert ici à se démarquer des conceptions molles de la société civile comme espace autonome.
} 
cherchent à impliquer c'est plutôt celui de la réévaluation de leurs rôles : dans quelle mesure seront-ils prêts à modifier leurs routines pour être actifs de la manière souhaitée et pour diversifier leurs interventions ? Deux réflexes structurels semblent s'y opposer. D'abord, ces partenaires de première ligne auraient tendance à déléguer aux spécialistes des violences conjugales une partie de leurs responsabilités, comme le déclarait - à propos des procureurs une dirigeante (Côté-victimes) au conseiller du ministre, à Jambes : " On les soulage quand on s'occupe de ça»-16/12/08). Ensuite, au sein de toutes les actions de dissémination envisageables, c'est surtout la formation qui les attire. Mais, ces professionnels de première ligne susceptibles d'être enrôlés comme partenaires, que disent-ils ? Si on les interroge en les considérant pour la place stratégique qu'ils occupent dans le premier cercle, puis en tant qu'ils auraient pu bénéficier d'actions de formation, fournissent-ils une réponse convergente et donc offrent-ils les mêmes prises de mobilisation aux promoteurs de la dissémination ?

\subsection{Les difficultés systémiques de l'intéressement}

Une enquête extensive menée en 2008-2009 auprès de représentants significatifs de ce premier cercle (deux parquetiers de référence ${ }^{16}$, deux intervenants sociaux de la police, un responsable de service d'aide aux justiciables, une criminologue dans une Maison de quartier) montre que les équipes des deux «côtés » et leurs principaux responsables sont bien identifiés et que leur action est approuvée, ce qui ne signifie pas nécessairement une bonne connaissance du cadre dans lequel il s'effectue (tous n'identifient pas « les Pôles ») et des difficultés à surmonter: l'intéressement ne suppose donc pas une adhésion fine à la problématisation $^{17}$.

\subsubsection{Les magistrats face à l'impératif d'une réponse dosée}

À certains égards, rencontrer des magistrats spécialisés, c'est s'adresser à des initiés en matière de violences conjugales et donc risquer d'obtenir une image faussée de la réceptivité de l'institution judiciaire - à tout le moins de l'institution de poursuite. En fait, cette spécialisation (le magistrat étant alors dit « de référence ») est toute relative et notamment liée à la structure de l'arrondissement judiciaire, ce qui a des effets en termes de catégorisation : "Il aurait été commode d'avoir un magistrat de référence, mais on doit faire plusieurs choses. On considère que ça fait partie de la délinquance urbaine (...) A Liège et à Charleroi, ils peuvent faire des économies d'échelle. Ici, il faut faire face aux violences urbaines ordinaires $^{18} \gg$. Il est en outre parfois difficile d'isoler les violences conjugales des autres faits délictueux ou criminels : "On est tenus de rassembler dans un seul dossier tous les faits qui concernent ces personnes ». Sauf s'il y a présomption de mise en danger d'enfants : "S'il y a violences à l'égard des enfants, c'est traité par la section délinquance urbaine avec mention à la section Jeunesse ». En conséquence, selon ce magistrat, "il est plus efficace d'avoir au sein $d u$ Parquet une section Famille ». On peut supposer dès lors que la sensibilisation du magistrat de service («Au niveau du Parquet, il y a un magistrat de service de garde 24h/24 et 365 jours par an. Ils sont censés me les [les cas de violences conjugales] rediriger ») aura une certaine incidence sur le traitement d'un signalement de violence intrafamiliale, même si selon notre interlocuteur le mécanisme semble fonctionner: "En fait dans le service de

\footnotetext{
${ }^{16}$ On a considéré ici les propos des deux magistrats (des substituts) - très convergents - comme formant un seul discours.

${ }^{17}$ Le courant de sociologie de la traduction autour de Michel Callon a plusieurs fois souligné - contre des applications mécaniques du modèle de l'innovation - que les quatre moments du processus ne sont pas des étapes irréversibles et que l'innovation est une action continuée : on l'aperçoit ici aussi.

${ }^{18}$ Ce premier extrait s'appuie sur la situation délégataire du tribunal de Mons par rapport à celui de Charleroi.
} 
garde, ça marche : les jeunes magistrats sont sensibles à ça» ${ }^{19}$. Nous sommes ici face à un premier enjeu pour l'innovation sociale dans un contexte qui voit se transformer progressivement le rôle du parquet de "chef de l'enquête" en "juge de l'enquête " (Cartuyvels, op . cit. : 541) : pour un magistrat de référence (ou sensibilisé) prêt à faire valoir les spécificités intrafamiliales d'un dossier de délinquance urbaine (donc: " enrôlé »), combien de magistrats qui en relativiseront la saillance ou la traiteront sur le mode de la singularisation (Bernheim, Commaille, 2012 : 295), donc : pas « intéressés » ${ }^{20}$ ?

Restent tout de même que les moyens d'agir (rappelés par la magistrate de Liège : mandat d'arrêt, libération sous conditions, médiation, rappel à la loi, sursis et suspension du prononcé - dans ces deux derniers cas avec mesure probatoire), sont subordonnés à la commission de l'infraction et à sa reconnaissance par l'institution (une classification, une graduation) : «Ma mission: rechercher crimes et délits, rapporter des preuves des infractions, poursuivre les auteurs (avec une instruction préparatoire). C'est la seule possibilité d'intervenir pour moi. Les menaces psychologiques ne sont pas des infractions. C'est un problème de qualification des faits. Il faut aussi un seuil de gravité». D'où la difficulté de la victimation pour les magistrats (et pour les policiers) : comment cumuler les "chiffres blancs» de la violence conjugale et intrafamiliale, les chiffres " gris » (infractions sans PV policier) et les chiffres «noirs : les femmes qui se font aider sans porter plainte ${ }^{21}$ ? L'attention des magistrats est, on l'a dit, attirée depuis 2006 sur la nécessité de traiter sans faille (un "principe d'intolérance, plutôt que de tolérance zéro » nuance un magistrat) cette partie émergée de l'iceberg et il a fallu un temps d'adaptation, des formations - et donc une variation selon les personnes qui est très inconfortable pour les innovateurs - avant de pouvoir en trouver les justes traductions: "Au début, il y a eu beaucoup de privations de liberté. Il a fallu se faire une échelle d'évaluation. Les ajustements sont dus aux formations et à ma propre sensibilisation ${ }^{22}$. Et parce que certains instruments comme la médiation pénale sont limités ${ }^{23}$ («En pratique, ça ne fonctionne pas bien : on l'utilise lorsqu'il y a maintien de la vie commune ») ou supposent des moyens (comme "la revisite »), il faut " essayer de trouver des solutions parallèles aux sanctions pénales », puisque «l'outil judiciaire n'est qu'un outil parmi d'autres ». Au fond, ce qui importe c'est qu'il y ait une réponse, même insatisfaisante, plutôt qu'une non-réponse qui semble concéder le droit à la récidive.

Sur ce point, la convergence des deux discours (celui des magistrats spécialisés et celui des Pôles) est forte, autour de l'idée d'incomplétude des réponses sectorielles : "Je n'apporte qu'une réponse partielle, qui doit être complétée ». Ce qui vaut pour l'action policière : «Il ne doit y avoir saisie du parquet que si ça apporte une plus-value par rapport à la police. Que le policier prenne ses dispositions, même si je préfère être avisé trop que trop peu »; ou pour les différents services auxquels il faut «passer le relais». L'incomplétude appelle le « réseautage » (terme souvent employé dans le groupe d'innovateurs que j'ai suivi) : "L'idée $d u$ réseau est souhaitable et opportune » me disait aussi un substitut.

Ces magistrats relèvent néanmoins certains obstacles figurant sur la route de la mise en

\footnotetext{
${ }^{19}$ Cette référence aux «jeunes » magistrats n'est pas une formule toute faite : si l'on en croit Cartuyvels (op. cit. : 543) émergerait en Belgique (et notamment en Communauté Francophone) une génération de magistrats plus sensibles au travail horizontal, à la multiplication des partenaires et donc moins tributaires de la law in the facts que tendrait à imposer l'autonomisation croissante du travail policier.

${ }^{20} \mathrm{Ch}$. Vanneste, qui note un «écart très important» entre la rhétorique de tolérance zéro et la réalité de la pratique, évalue à 34\% la part de prévenus pour lesquels la procédure aurait pu être poursuivie au regard cette politique et qui ne l'a pas été (Vanneste, op. cit., résumé : 6).

${ }^{21}$ Ces problèmes d'enregistrement semblent être à la source des variations, selon les arrondissements, des taux de signalement policiers comme des taux d'affaires enregistrées (Vanneste, op. cit. : 46-49).

${ }^{22}$ L'énoncé du champ d'application du principe est considéré comme un facteur décisif (Vanneste, op. cit., résumé : 7-8).

${ }^{23}$ Cela représente 7\% des prévenus dont l'infraction a été établie (Vanneste, op. cit. : 75). Voir : Mincke, 2012.
} 
réseau. Obstacles professionnels d'abord. Ce sont ceux des premières lignes médico-sociales : «le secret professionnel partagé, c'est le monstre du Loch Ness. L'objectif est de faire cesser un danger, pas forcément de poursuivre. Je ne suis pas frileux par rapport à ça, mais les médecins et les intervenants sociaux le sont». Ceux des policiers : "L'indice $42^{24}$ : une catégorie fourre-tout de différends familiaux qu'ils peuvent utiliser au lieu de ne pas faire de $P V »$. Mais aussi, obstacles institutionnels: "la coordination rencontre un problème d'échelle, car la Province n'est pas un échelon utile. Il faudrait travailler par zones de police (...) et renforcer les A.S. et psycho' pour faire de la première ligne au sein de la police, en privilégiant un principe d'individuation, un cas par cas, qui ferait voir quel est le partenaire pertinent $\gg$.

Ces intentions se heurtent aussi au principe de réalité de l'institution judiciaire : masse de dossiers, tendance à la fermeture sur les routines, lenteur de réaction, persistance de formes déguisées de classement sans suite (Lenoir, Gautron, 2014), glissement vers la délégation au travail policier. Les activistes doivent donc comprendre que le volontarisme a ses limites: "Notre problème à nous : on doit faire face à la masse, tandis que Praxis a besoin d'une décision du tribunal ou d'un engagement personnel, soit : quelques dossiers sur la masse ». Les essais de réunions d'information pour les magistrats ayant connu peu de succès (et de ce fait fortement mécontenté le procureur), un substitut dit n'avoir "pas renouvelé l'expérience ». Le contact direct a parfois plus de chances d'aboutir: "Praxis essaie de toucher les juges. Il faut leur rappeler les possibilités ».

On comprend que les deux substituts aient une appréciation élogieuse du travail effectué par les équipes du Pôle auprès de leurs partenaires ("une collaboration remarquable»), notamment, la mise en réseau. C'est aussi le cas pour le travail effectué sur leur terrain propre (« un travail magnifique auprès des victimes »). Comme on va le voir, c'est à peu près dans les mêmes termes que les autres membres - que l'on présentera ici en bloc - de ce réseau en devenir jugent ceux qui entendent les mobiliser après les avoir intéressés et enrôlés.

\subsubsection{Prudence et attentes limitées chez les autres partenaires}

Les trois personnes que j'ai interrogées au sein de la police représentent un point de vue spécifique : ce sont des intervenants sociaux qui ont à faire face au moins autant aux préjugés concernant les travailleurs sociaux qu'à ceux que peuvent déclencher certaines victimes ou certains auteurs. D'emblée, on saisit que les coopérations seront limitées et que la formation apparaît au contraire comme la ressource permettant de faire avancer un peu les mentalités de leurs collègues.

«On n'est pas policières, on doit avoir du doigté, car beaucoup de policiers gardent leurs a priori sur les travailleurs sociaux» (criminologue, Police, Région de Liège) : pour les deux personnes (et une stagiaire) rencontrées en région liégeoise, la pertinence du Pôle semblait se définir au niveau des formations (approuvées et redemandées), mais un peu moins au niveau des coopérations directes police / Pôle sur le terrain (une des personnes citant même l'exemple d'une « mauvaise collaboration entre policiers et le CVFE» du fait des policiers). Une coopération directe adéquate avec les associations spécialisées dépend pour l'instant beaucoup trop de la masse des affaires mêlées, dont certains policiers s'occupent mieux que d'autres («X... c'était l'oiseau rare, mais il n'est plus là »).

L'assistante sociale de la police, déjà formée sur ces questions et engagée avec deux autres professionnelles de la ville dans des projets ciblés, s'est montrée la plus précise sur l'usage qu'on peut faire, au sein de la police, des formations reçues. Il s'agissait pour elle d'approfondir (notamment sur la question des enfants) pour être un relais d'information plus

\footnotetext{
${ }^{24} \mathrm{NB}$ : Cet entretien date de fin 2008. L'indice générique « 42 » n'est plus utilisé aujourd'hui : il a été fragmenté (exemples : $42 \mathrm{~L}$ - différend familial ; $42 \mathrm{O}$ - enfance en danger).
} 
efficace au sein de la police où «c'est toujours à recommencer. Ils sont blasés et en ont marre d'en entendre parler. Mais pour nous aussi, dans quelques années, il faudra recommencer... » (A.S., police région de Liège). Elle partage le point de vue de sa collègue de la Maison de Quartier, qui estime avoir reçu, notamment de la part des binômes agissant au nom des Pôles, une formation de qualité : «L'un plus pratique, l'autre plus théorique : c'était complémentaire. On voit que c'était pas de l'improvisation» (criminologue, Maison de Quartier, région de Liège). Elle se dit d'ailleurs persuadée de l'opportunité de la mise en place d'un groupe de travail et d'une supervision.

En bref, je n'ai pas eu le sentiment d'une attente importante en direction du Pôle, comme si un traitement intersectoriel de ces questions était écrasé par la masse des dossiers ou par les effets de mode, sans parler des incertitudes concernant les décisions des parquetiers : " $A u$ début, c'était tout feu tout flamme. Ça retombe aujourd'hui. Ce qui nous inquiète, c'est la réaction du Parquet, car les parquetiers ne sont pas tous convaincus et mettent parfois les policiers en porte-à-faux» (criminologue, Police, région de Liège).

Pour d'autres partenaires situés en première ligne l'avis est à peu près le même. On ne voit pas poindre de collaboration directe entre première et seconde ligne, mais plutôt une demande d'outils : la criminologue rencontrée à la Maison de Quartier était surtout à la recherche d'une sensibilisation pratique à la détection des violences conjugales ayant des répercussions sur les enfants : «j'ai maintenant des instruments pour réagir ».

Autre type de partenaire potentiel, situé en première ligne, mais avec des missions plus larges que la précédente : le SASJ. Un directeur fait remarquer que les occasions de coopération ne sont pas encore monnaie courante ou qu'elles n'ont pas été approfondies : "Praxis travaille à la fois sur mandat et à la demande. Dans notre service, il y a la possibilité de faire une absorption d'une partie de la demande. On l'a fait savoir à Praxis, mais on n'est pas allé plus loin ». Notre interlocuteur se définit comme "complémentaire du travail fait par Praxis", alors que «le CVFE a une équipe plus importante et donc gère tous types de situations sans faire appel à nous » (idem).

Pourtant, selon cette personne, les choses progressent : parfois grâce à l'opiniâtreté de certains («la ténacité d'A.B. /Procureur emblématique de Liège, qui a quitté la juridiction/»), mais plus généralement parce qu'il y a «la volonté politique de prendre en charge » (idem). Certes "le paysage se complexifie» (idem), mais ce directeur de SASJ ne définit pour l'instant aucune attente d'une mise en réseau du type "Pôle», paraissant se satisfaire de «l'interconnaissance» produite par «les réunions mensuelles au sein de la Commission provinciale $\gg($ idem $)$.

\subsubsection{Des formes d'intéressement par le flou ?}

On voit les limites de l'intéressement et de l'enrôlement, y compris de ce premier cercle : à l'exception des magistrats de référence (cela ne vaut donc pas pour tous les parquetiers), les partenaires potentiels tendent à se satisfaire d'apports cognitifs (donc un surcroît de formation peut y répondre) et restent prudents quant à la mise en mouvement de l'équipement dans son ensemble $^{25}$. Est-ce une forme d'échec de la dissémination (une forme de réintroduction des spécialistes)? Au contraire, ces décalages peuvent-ils être incorporés au dispositif d'enrôlement? Allant dans ce dernier sens, un magistrat paraissait craindre que le succès du processus de coopération «inter-lignes » n'entraîne une sorte de déresponsabilisation des professionnels qui deviendraient des éléments d'un dispositif tentaculaire faisant circuler les

\footnotetext{
${ }^{25}$ Mais l'intention d'avancer vers un degré de politisation (au sens défini ici) plus fin ne peut être mise en doute : le Ministre semble avoir sollicité le Collège des Procureurs Généraux pour obtenir une note-cadre sur la manière dont les Parquets pourraient offrir une approche multidisciplinaire des actions en direction des violences conjugales.
} 
justiciables entre spécialistes - un réseau devenu une toile d'araignée (AUTEUR, a). Or, selon lui, les jeunes magistrats doivent "prendre leurs dispositions », c'est-à-dire assumer la prise d'initiative en situation sans se reposer sur les spécialistes ou les policiers. Autrement dit, l'esprit même du dispositif d'enrôlement et un affinement de la justice restaurative.

Cette percée restreinte du paradigme minoritaire de la RPM peut-elle être attribuée à une tectonique interne aux continents judiciaire, policier ou social ou bien est-elle reliée à la problématisation des innovateurs? Il est difficile de trancher nettement. Mais c'est précisément cette difficulté qui fait la force des dispositifs d'intéressement et d'enrôlement : ils peuvent fonctionner comme objets-frontières (Trompette, Vinck, 2009) ou, dans un autre vocabulaire, comme malentendus fonctionnels (Boussard et al., 2004). Chacun peut participer aux actions sans avoir à faire toute la clarté sur les implications ultimes des conceptualisations, y trouver son intérêt avec ses propres connotations et à tout le moins jouer un rôle, celui qui autorise des formes de mobilisation. Les débats très sensibles sur la dangerosité - déjà mentionnés plus haut - au sein des équipes sont à cet égard dans une situation de décalage «intéressante» avec ceux qui agitent la sphère politique ou universitaire.

\subsection{La formation : les premiers pas de la mobilisation}

En 2009, les Pôles de ressources de Liège et La Louvière ont été officiellement répertoriés par la R.W. comme organismes privilégiés de formation spécialisée en approche des violences conjugales et intrafamiliales à destination de tous les professionnels de première ligne. En 2010, la Région a dressé une liste des services concernés et fixé le nombre d'heures dont ils pourront bénéficier. Cette reconnaissance d'une compétence, au moins en matière de formation n'a pas manqué de faire quelques mécontents et de conduire à des rééquilibrages ultérieurs, mais c'est une étape cruciale dans le déroulement de l'innovation : au moins sur le plan de la formation, le dispositif va au-delà de l'enrôlement, c'est-à-dire jusqu'à la phase de mobilisation de «porte-paroles» qui diffusent dans l'espace public les problématisations jusqu'alors confinées aux sphères d'influence directe des organisations.

\subsubsection{Situation d'une forme emblématique de dissémination}

La formation est l'une des activités les plus reconnues des Pôles, même si ses animateurs craignent que les instances régionales ne cantonnent leur soutien à cette seule dimension. D'une part, elle est à la fois l'action la plus tournée vers l'extérieur et, dans un univers où la ressource experte demeure rare, la plus sollicitée; d'autre part, elle n'a pas entretenu de tensions entre équipes et a donc pu être très rapidement stabilisée, voire pratiquement modélisée.

Deux modalités sont disponibles : d'abord, un programme de base de trois journées, conçu pour acquérir des repères, grâce à un travail sur des illustrations concrètes de situations de violences intrafamiliales; ensuite, des journées complémentaires. Il ne s'agit pas, pour les organisateurs, de céder à la demande récurrente des professionnels de première ligne de disposer d'un kit d'intervention qui écraserait la spécificité des situations et qui les mettrait, sans qu'ils l'aient cherché, en position de référent unique : "Les stagiaires de première ligne sont souvent "poussés vers le stage" par leurs équipiers sur un mode délégataire, avec pour visée plus ou moins avouée d'en faire ensuite la personne de référence vers laquelle le reste de l'équipe dirige tout le flux, sans se sentir concernées ou compétente. C'est le paradoxe du retour: le stagiaire a l'impression d'être encore moins bien armé pour faire face aux problèmes qu'avant son stage, puisqu'il est désormais dépositaire supposé d'un savoir. Certes, il a besoin d'être soutenu, mais il doit revenir chez lui avec une modalité de "travail 
isole”" (...)» (Rapport, 2009). On voit ici la minceur de la frontière entre le «porte-parole » (un médiateur qui dissémine l'innovation) et le «référent» (un intermédiaire qui filtre les savoirs).

Chaque formation est confiée à des "dyades intersectorielles de formateurs » (une personne du côté-victimes, l'autre du côté-auteurs), auxquelles il est demandé d'avoir cinq ans d'expérience de l'intervention spécifique et deux ans d'expérience de coopération intersectorielle. Cette exigence pourrait conduire à spécialiser à l'excès des salariés comme formateurs, c'est pourquoi les Pôles se sont efforcés d'organiser un roulement.

On a vu plus haut quel était le public électif en termes professionnels (les magistrats, les policiers et les travailleurs sociaux), auquel il faut adjoindre, en termes d'institutions cette fois, les Maisons d'accueil et plus généralement les Plates-Formes d'Arrondissement. Le tableau 1 (établi à partir des Rapports 2009 et 2010) donne un aperçu du public effectivement accueilli en 2009 et 2010. On notera que les magistrats en sont absents : les magistrats de référence et les parquetiers les plus aguerris avaient en général fait partie des promotions précédentes, ce qui laisse entière la question de la sensibilisation des jeunes parquetiers, des magistrats non spécialisés et des juges.

Tableau 1

La participation des intervenants de première ligne aux sessions de formation

\begin{tabular}{|l|l|r|r|}
\hline Répartition professionnelle ou fonctionnelle & Année & $\begin{array}{c}\text { Nombre de } \\
\text { participants }\end{array}$ & $\begin{array}{c}\text { Nombre } \\
\text { d'heures }\end{array}$ \\
\hline Policier(e)s & 2009 & 17 & 132 \\
\hline Intervenants sociaux (secteur public) et judiciaires* & 2009 & 134 & 408 \\
\hline Intervenants sociaux (secteur privé)** & 2009 & 44 & 132 \\
\hline Enseignant(e)s (secteur privé)*** & 2009 & 31 & 96 \\
\hline Salarié(e)s des Maisons d'Accueil $* * * *$ & 2010 & 42 & 252 \\
\hline Participant(e)s aux plates-formes d'arrondissement***** & 2010 & 205 & 468 \\
\hline Ecoutant(e)s (Ligne d'écoute téléphonique) & $2009-10$ & 21 & 318 \\
\hline Total & & 494 & 1806 \\
\hline
\end{tabular}

*Arrondissements de Verviers, Liège, Huy, Eupen et Province de Luxembourg

** Bruxelles, Namur, Nivelles, La Louvière

***Bruxelles et Namur

**** Ensemble de la Région Wallonne

***** Tournai, Mons, Charleroi, Namur et Dinant

Quoi qu'il en soit, cette revue d'effectifs et de temps de formation montre l'ampleur de l'action entreprise et l'écho qu'elle a reçu : en deux ans, près de 500 personnes pour plus de 1800 heures de formation.

\subsubsection{Réception et appropriation de l'offre}

En 2011, j'ai eu la possibilité d'enquêter auprès de personnes ayant suivi certaines de ces formations (des entretiens semi-directifs sur deux échantillons restreints construits à partir de participants aux formations en 2010 ; voir tableau 2). Une analyse thématique approfondie des réponses permet de dégager plusieurs résultats significatifs.

Tableau 2

Répartition des personnes interrogées

par lieu d'exercice et par fonction exercée

\begin{tabular}{|l|l|r|r}
\hline Catégories & Institutions d'exercice & $\mathbf{n}$ & Professions ou fonctions exercées
\end{tabular}




\begin{tabular}{|l|l|r|l|r|}
\hline & \multicolumn{1}{|c|}{ des personnes interrogées } & & \multicolumn{1}{|c|}{ par les personnes interrogées } & \\
\hline $\begin{array}{l}\text { Membres } \\
\text { AMA }\end{array}$ & Maisons d'accueil & 6 & $\begin{array}{l}\text { Psychologue (2); Assistant social (4); } \\
\text { Educatrice (1); Coordinateur (1) }\end{array}$ & 8 \\
\hline $\begin{array}{l}\text { Participants aux } \\
\text { Plates-formes }\end{array}$ & $\begin{array}{l}\text { Hôpital ; SAPV ; SAJ ; Maison } \\
\text { de Justice ; CPAS ; Femmes } \\
\text { Prévoyantes Socialistes }\end{array}$ & 7 & $\begin{array}{l}\text { Psychologue (3); Assistant social (3); } \\
\text { Assistante de Justice (2); Animatrice } \\
\text { socio-culturelle (1) }\end{array}$ & 9 \\
\hline Total & & 13 & Dont : 15 femmes et 2 hommes & 17 \\
\hline
\end{tabular}

Un premier enseignement: les participants sont impliqués et venus sans contrainte d'engagement (ou on profite de l'offre ou on la sollicite). L'ensemble des répondants est directement concerné par les violences conjugales : pour une partie c'est une préoccupation émergente (une personne veut " essayer de comprendre ce qui se passe »), pour la moitié une fonction professionnelle. Pour la plupart (11 sur 17) ils savent déjà à qui ils vont avoir affaire et cette information préalable semble crédibiliser la formation: en majorité, c'est la présentation symétrique des violences conjugales (une personne «voulait absolument connaître le $P D C »)$ qui est recherchée et singulièrement, un meilleur éclairage sur les auteurs : quelqu'un évoque des collègues qui auraient pu venir «si on avait su » que c'était ça.

La satisfaction, générale s'appuie sur des repères relativement cohérents : pour près de la moitié, il s'agit de la pratique par «les binômes » du "langage commun » propre au modèle d'analyse, mais aussi la méthode de travail pratiquée par les animateurs. Le PDC n'est pas toujours clairement nommé, mais c'est bien son rôle d'équipement transposable, plus précis que le « cycle de la violence », qui est retenu par les répondants.

À l'exception d'une petite minorité, ils pensent pouvoir mettre en œuvre l'outil reçu en formation, mais ils n'en revendiquent pas la maîtrise technique : les formés estiment pouvoir désormais porter une attention systématique à ce qui avant n'avait l'air que de détails. Les quelques difficultés soulignées montrent que les obstacles à la dissémination sont rattachés par les répondants à des spécificités de terrain : 1/pour certains, le PDC semble fait pour d'autres terrains (exemple: pour le travail avec les victimes; pour le travail de dévictimisation mais pas de réinsertion) ou trop général ; 2/le travail "en première ligne » signifie souvent «un manque de temps » et une difficulté «à faire la part des choses »; 3/la "spécificité » de la probation / réinsertion réclamerait d'autres instruments de travail, "plus d'apports techniques », mais demanderait aussi de "sensibiliser les magistrats »; 4/enfin, en Maison d'Accueil, on ne sait jamais quand les victimes partiront, ce qui demande de savoir réagir rapidement et bloque le travail de long terme.

Les ajustements demandés sont mineurs, mais offrent des occasions, pour les Pôles, de témoigner d'une politique participative (ne pas seulement apporter des ressources, mais mobiliser les expériences des participants) et d'infléchir leurs axes de travail pour : 1/ faire naître une demande concernant le travail en équipe dans les institutions d'exercice (comment réutiliser les contenus de formation dans un travail avec des collègues qui n'y ont pas participé ?) ; 2/ faire baisser les attentes en termes de « choses à savoir » sur les violences conjugales ; 3/ faire monter les ambitions en termes de gestes professionnels participatifs (polyvalence, intervention en équipe et en réseau).

Ces intentions sont en cohérence avec tout ce qui vient d'être dit à la fois au niveau global (la pertinence politique, judiciaire et professionnelle du travail de dissémination dans l'espace social qui s'est construit autour de la thématique des violences conjugales) et au niveau plus restreint de la formation (la pertinence de l'accent mis sur la formation comme première étape d'une appropriation des outils d'intervention par des personnels de première ligne). Toutefois, on aura pu apercevoir, au cours de l'examen de la réception de cette offre, plusieurs difficultés de mise en œuvre qui incitent à s'interroger sur la portée et les limites de cette 
innovation au regard du changement à apporter au système d'intervention judiciaire, policier et social.

\section{Des prises sociales dans les plis du droit}

L'originalité du dispositif d'intervention sur les violences conjugales qui vient d'être présenté aurait permis plusieurs approches, à commencer par l'analyse du travail avec ses destinataires finaux (auteurs et victimes). J'ai privilégié un aspect à première vue moins significatif de la prise en charge de ce qui est devenu une cause publique : le processus de transfert d'une partie du geste professionnel spécialisé à des non-spécialistes. Il m'a semblé que son caractère innovant avait des répercussions sur l'ensemble du traitement de ce problème. Mais plus que l'apport d'un éclairage sur de nouvelles pratiques de coopération, l'intérêt de cet angle d'attaque est selon moi de faire apparaître les démarquages structurels et conjoncturels qui offrent un espace à des dispositifs d'intervention souvent considérés comme le fruit de l'urgence. Là est la dimension ontologique : l'être-là du fait coopératif est inscrit dans une trame qui le fonde. Ce qui pourrait n'être qu'une simple préoccupation du sociologue est aussi au centre du souci des innovateurs qu'il a suivis. C'est parce que ces activistes questionnent leur rapport à l'institution judiciaire (et à ses appuis policier et secourable) qu'ils problématisent l'efficience d'une mobilisation des professionnels se trouvant au premier contact des violences conjugales (les «premières lignes »). Ce questionnement les incite aussi à construire un dispositif d'intéressement au sein duquel leurs partenaires potentiels pourraient déplacer leurs rôles habituels. Cette réflexivité les conduit enfin à soutenir notamment par de la formation - les initiatives inusitées des magistrats, des policiers et des travailleurs sociaux qui sont comme les premiers frémissements de l'impact sociétal de leur problématisation.

Le pari de cet article est de tirer parti de l'analyse d'une innovation qui redéfinit le travail des intervenants spécialisés avec le système judiciaire et de la traiter comme l'une des émergences de la dialectique du droit et de la structure sociale. Une étude complémentaire serait nécessaire pour sociologiser ces interrogations sur un autre plan, par une prise en compte à la fois des composantes sociodémographiques et du rapport aux normes des magistrats (permettant de comprendre leur «mode d'emploi des institutions », comme le préconisaient il y a longtemps Faugeron et Jakubowicz,1984:660), si du moins on veut échapper à la fatalité des explications par le poids des personnalités charismatiques ou des réseaux d'influence.

Quant aux effets visibles de l'émergence d'un dispositif d'alliance du social et du judiciaire, ils demeurent restreints : cette innovation - un processus interminable scandé par des moments décisifs - n'a pas atteint un cliquet d'irréversibilité. De nombreux chantiers sont encore à l'état d'ébauche et une certaine usure dans le travail intersectoriel est constatable. L'intéressement et l'enrôlement ne sont pas seulement des défis pour les partenaires, mais aussi pour les équipes elles-mêmes, toujours confrontées à l'impératif de réflexivité. Les innovateurs seront-ils disposés à accepter que leur machinerie n'atteigne son but que de manière imparfaite, comme on les en a supposés capables ? Plusieurs dirigeants reconnaissent que la première ligne ne peut pas tout faire, en particulier parce qu'elle manque de moyens pour estimer la dangerosité : ce constat est à la fois une justification du travail de dissémination par la formation et le signal que, même en cas de succès du dispositif, les associations ne peuvent abandonner leur propre travail de première ligne.

Malgré ces limites, l'interpellation des différents intervenants du champ des violences conjugales à laquelle procèdent ces agitateurs a quelques chances d'être entendue. Peut-être même à cause de ces limites, qui éloignent le risque du dispositif tentaculaire tout en préservant, par la présence d'objets-frontières (la sécurité, la dangerosité, la justification, 
l'empowerment), la force de ce dispositif faible : celle qui fait coopérer sans prérequis. Mais, de l'avis même des principaux animateurs, le chemin est encore long, les enthousiasmes retombent vite et le soutien politique est vécu comme inconstant, en tout cas sur le plan financier.

Dès lors, la revendication du «pragmatisme» (entendu au sens courant de pratique immédiatement efficace) est-elle devenue, ici comme en de nombreux autres champs, l'alpha et l'oméga de l'intervention? On a vu que les protagonistes pouvaient se satisfaire d'un pragmatisme procédural dans le domaine judiciaire (peut-être même d'un pragmatisme judiciaire tout court) sans attendre l'installation solennelle d'une nouvelle rationalité juridique, du moins tant que l'effet de contagion des principes de justice restauratrice se fera sentir. Si le couple droit / violences conjugales reste de multiples façons « un couple à problèmes " sur le plan théorique, les innovateurs que nous avons suivis ont réussi à se donner des prises solides pour avancer sans attendre dans les plis du droit, comme ils l'ont fait jusqu'ici dans les méandres du champ politique, en repoussant l'interrogation sur la judiciarisation, pour promouvoir une politique de l'implication. Et peut-être n'y a-t-il de réponse à ce « problème de couple » que de ce type, c'est-à-dire partielle.

\section{Addendum}

Cet article a été relu par quatre des militants concernés, que je remercie ici. Outre la correction de quelques erreurs factuelles, cette relecture offre une suite au processus d'innovation qui semble valider l'hypothèse de la force des dispositifs faibles. En 2017, si les binômes de formation continuent, le travail intersectoriel semble en panne. Mais les trois associations assurent ensemble la tenue de la ligne téléphonique wallonne dévolue aux violences conjugales. L'histoire progresserait-elle une fois de plus «par son petit côté »?

\section{Références}

AUTEUR, a, b, c, d.

Akrich M., Callon M., Latour B., 2006, Sociologie de la traduction. Textes fondateurs, Paris, Presses des Mines de Paris.

Ayotte R., Brisson M., Potvin P., Prud'homme D., Tremblay D., 2007, La légitimité du pouvoir chez les conjoints dominants: une étude exploratoire des stratégies de justification du modèle du Processus de Domination Conjugale (PDC), rapport de recherche pour la Direction de la santé publique de la Mauritie et du Centre-du-Québec, L'Accord Mauritie.

http://www.accordmauricie.com/Pdf/RechercheLaLegitimiteDuPouvoir.pdf

Begon R., 2007, Violences conjugales, de la sphère privée à la pénalisation, document, Liège, Collectif contre les violences familiales et l'exclusion (CVFE). http://www.cvfe.be/sites/default/files/doc/EP2007-ReneB-VCetviepriv.pdf

Bernheim E., Commaille J., 2012, Quand la justice fait système avec la remise en question de l'État social. Présentation du dossier, Droit et société, 81-2, 281-298.

Born M., Glowacz F., 2006, Pour que les violences domestiques soient sans avenir, Revue Internationale de Criminologie et de Police Technique et Scientifique, January 2006, 4, 387-297.

Boussard V., Mercier D., Tripier P., 2004, L'aveuglement organisationnel. Ou comment lutter contre les malentendus, Paris, CNRS Editions.

Cador P., 2005, Le traitement juridique des violences conjugales: la sanction déjouée, L'Harmattan - Logiques sociales.

Cardi C., Devreux A.-M., 2014, Le genre et le droit: une coproduction, Cahiers du genre, $\mathrm{n}^{\circ} 57,5-18$. 
Cartuyvels Y., 2004, Police et parquet en Belgique : vers une reconfiguration des pouvoirs?, Droit et société, 58-3, 523-542.

Cavaillès J., 1960, Sur la logique et la théorie de la science, Paris, P.U.F. (1 è édition : 1947).

Chateauraynaud F., 2011, Argumenter dans un champ de forces. Essai de balistique sociologique, Paris, Editions Petra, coll. « Pragmatismes ».

Cauchie J.-F., Kaminski D., 2007, Éléments pour une sociologie du changement pénal en Occident. Eclairage des concepts de rationalité pénale moderne et d'innovation pénale, Champ pénal/Penal field [En ligne], Vol. IV, URL : http://champpenal.revues.org/613 ; DOI : 10.4000/champpenal.613

Commaille J., 2010, Les vertus politiques du droit. Mythes et réalités, Droit et société, 76-3, 695-713.

Cresson G., 2002, Médiation familiale et violence conjugale, Cahiers du Genre, 2/2002 (n ${ }^{\circ}$ 33), p. 201-218. URL : www.cairn.info/revue-cahiers-du-genre-2002-2-page-201.htm. DOI : $10.3917 /$ cdge.033.0201.

Danet J., 2008, La dangerosité, une notion criminologique, séculaire et mutante, Champ pénal/Penal field [En ligne], Vol. V|2008. URL : http://champpenal.revues.org/6013 ; DOI : $10.4000 /$ champpenal.6013

Delage P., 2015, La violence conjugale à l'épreuve de l'État social : une comparaison francoétats-unienne, Enfances, Familles, Générations, $\mathrm{n}^{\circ}$ 22, 68-84. URI : http://id.erudit.org/iderudit/1031119ar. DOI: 10.7202/1031119ar

Dubois C., 2008, Restauration et détention en Belgique : genèse de la circulaire ministérielle du 4 octobre 2000, Droit et société 2008/2 (nº 69-70), 479-505.

Faget J., 2004, Médiation et violences conjugales, Champ pénal/Penal field [En ligne], Vol. I | 2004. URL : http://champpenal.revues.org/50 ; DOI : 10.4000/champpenal.50

Faugeron C., Jakubowicz P., 1984, Les magistrats et la loi pénale, Revue française de sociologie, XXV-4, 658-683.

Garapon A., 1991, L'idée de droit social : G. Gurvitch, in P. Bouretz (dir.), La force du droit. Panorama des débats contemporains, Paris, Editions Esprit, 215-228.

Goulet F., Vinck D., 2012, L'innovation par retrait. Contribution à une sociologie du détachement, Revue française de sociologie, Vol. 53-2, 195-224. DOI $10.3917 / \mathrm{rfs} .532 .0195$

Hegel G.W.F., 2013, Principes de la philosophie du droit, trad. J.-F. Kervégan, Paris, PUF.

Herman E., 2011, Idéal féministe et injonction à l'autonomie économique. Le travail social des associations luttant contre les violences conjugales, Pensée plurielle 2011/1 ( $\mathrm{n}^{\circ} 26$ ), 81-92. DOI 10.3917/pp.026.0081

Herman E., 2012, Paradoxes du travail social au sein des associations de lutte contre les violences conjugales, Informations sociales 2012/1 ( $\left.\mathrm{n}^{\circ} 169\right), 116-124$.

Lenoir A., Gautron V., 2014, Les pratiques des parquets face à l'injonction politique de réduire le taux de classement sans suite, Droit et société, 88-3, 591-606.

Luhmann N., 1989, Le droit comme système social, Droit et société, n¹1-12, 53-67 ; DOI : 10.3406/dreso.1989.1024; 33621989 num 1111024

http://www.persee.fr/doc/dreso_0769-

Mary P., Kaminski D., Maes E., Vanhamme F., 2011, Le traitement de la "dangerosité" en Belgique : internement et mise à la disposition du gouvernement, Champ pénal/Penal field [En ligne], Séminaire du GERN "Longues peines et peines indéfinies. Punir la dangerosité" (2008-2009). URL : http://champpenal.revues.org/8351

Milburn P., Kostulski K., Salas D., 2010, Les procureurs. Entre vocation judiciaire et fonctions politiques, Paris, PUF.

Mincke C., 2006, La proximité dangereuse. Médiation pénale belge et proximité, Droit et société 2006/2 (n63-64), 459-487. 
Mol A.-M., 1999, Ontological Politics. A Word and Some Questions », in J. Law, J. Hassard, Actor-Network Theory and After?, The Sociological Review Monographs, Volume 47, Issue S1, 74-89.

Pêcheux C., 2012, Violences conjugales: où en est la Belgique à l'heure actuelle? http://www.amnesty.be/je-veux-m-informer/nos-blogs/archives/le-blog-de-clairepecheux/article/violences-conjugales-ou-en-est-la

Supiot A., 2010, L'esprit de Philadelphie. La justice sociale face au marché total, Paris, Seuil.

Teubner G., 1987, L'ordre social par le "bruit législatif"? La fermeture autopoïétique comme système de régulation juridique, Archives de philosophie du droit, Paris, Sirey, tome 32, 249-275.

Thelen K., 2003, Comment les institutions évoluent: perspectives de l'analyse comparative historique, L'Année de la régulation, $\mathrm{n}^{\circ} 7,13-44$.

Trompette P., Vinck D., 2009, Retour sur la notion d'objet-frontière, Revue d'anthropologie des connaissances, Vol. 3, $\mathrm{n}^{\circ}$ 1, 5-27. URL : www.cairn.info/revue-anthropologie-desconnaissances-2009-1-page-5.htm. DOI : $10.3917 /$ rac.006.0005.

Vanneste C., 2016, La politique criminelle en matière de violences conjugales: une évaluation des pratiques judiciaires et de leurs effets en termes de récidive, rapport de recherche et résumé, Bruxelles, Direction Opérationnelle de Criminologie, $\mathrm{n}^{\circ} 41$.

Vidal C., 2008, Wat is een wereldbeeld? (What is a worldview ?), in Van Belle, H. \& Van der Veken, J., Editors, Nieuwheid denken. De wetenschappen en het creatieve aspect van dewerkelijkheid, Leuven, Acco.

Vigour C., 2004, Réformer la justice en Europe. Analyse comparée des cas de la Belgique, de la France et de l'Italie, Droit et société 2004/1 (n56-57), 291-323.

Vigour C., 2009, Politiques et gouvernements fédéraux en Belgique, entre contraintes coalitionnelles et logique de compromis, Politix, 88-4, 63-86. URL: www.cairn.info/revue-politix-2009-4-page-63.htm. DOI : 10.3917/pox.088.0063.

Walque (de) G., 2008, Lutte contre les violences faites aux femmes : une question de santé publique!, rapport, Bruxelles, Institut Emile Vandervelde - www.iev.be - iev@iev.be 